\title{
DIFFUSION LIMIT OF A HYPERBOLIC SYSTEM WITH RELAXATION
}

\author{
Shi Jin and Hailiang Liu
}

\begin{abstract}
In this paper, we introduce a diffusive scaling to a hyperbolic system with relaxation and prove that, under such a scaling, the solution converges to that of a nonlinear convection-diffusion equation. Using energy estimates, such a limit is justified with the initial data prescribed around a traveling wave solution of the relaxation system.
\end{abstract}

\section{Introduction}

Hyperbolic systems with relaxation arise in a wide variety of physical problems, ranging from linear and nonlinear waves [22], kinetic theory [3], to multiphase and phase transition modeling. In these problems, usually one cannot find the exact solution. The behavior of the solution can be understood in some asymptotic regimes.

In recent years, much attention has been paid to the study of the zero relaxation limit. In this regime, a complicated system may asymptotically be replaced by a much simpler hyperbolic system, the behavior of the latter is either already well understood or easier to analyze. Rigorous justification of such a limit has been made by Liu [15], Chen et al. [5], Collet and Rascle [6], and Natalini [18] for $2 \times 2$ systems, where the zero relaxation limit is a scalar conservation law, using compensated compactness or bounded variation (BV) estimates. Such results were also obtained for multidimensional relaxation systems that converge to scalar equations by Katsoulakis and Tzavaras [9] and Natalini [19]. See [20] for a survey of recent results in this direction. More recently such a program has been carried out for relaxations to hyperbolic systems by Serre [21].

In this article, we study a different asymptotic limit, which leads from a hyperbolic system with relaxation to a convection-diffusion equation. The proper scaling of this asymptotic regime, besides the usual assumption of a small relaxation time $\varepsilon$, requires a long time and weak nonlinearity assumption. This kind of long time behavior is important physically. Among the famous examples are the transport equation in diffusive regimes $[2,10]$ and the Boltzmann equation near incompressible Navier-Stokes regimes $[4,1,12]$. For hyperbolic systems with relaxation, various diffusion limits have been investigated $[5,7,16,17]$, but the asymptotic scaling studied in this article is new to our best knowledge.

We begin with the hyperbolic system with relaxation constructed by Jin and Xin [8]:

$$
\begin{aligned}
u_{t}^{\varepsilon}+\tilde{v}_{x}^{\varepsilon} & =0, \\
\tilde{v}_{t}^{\varepsilon}+a u_{x}^{\varepsilon} & =-\frac{1}{\varepsilon}\left[\tilde{v}^{\varepsilon}-f\left(u^{\varepsilon}\right)\right]
\end{aligned}
$$

Received April 22, 1997, revised July 18, 1998. 1991 Mathematics Subject Classification: 35L45.

Key words and phrases: hyperbolic system, relaxation, diffusion limit, energy estimate. 
where $\varepsilon>0$ is the relaxation time. This system was introduced as a new way of regularizing the hyperbolic system

$$
u_{t}+f(u)_{x}=0 \text {. }
$$

In fact, for small $\varepsilon$, using the Chapman-Enskog expansion, one may get the convectiondiffusion equation,

$$
u_{t}+f(u)_{x}=\varepsilon\left(\left(a-f^{\prime}(u)^{2}\right) u_{x}\right)_{x}
$$

which gives the viscosity solution to (1.2) if the following subcharacteristic condition holds:

$$
a>f^{\prime}(u)^{2} .
$$

It has been proved that the solution of (1.1) does converge to the entropy solution of $(1.2)[5,6,18]$. Thus, (1.1) provides a physically natural way to regularize (1.2), parallel to the regularization of the Euler equation by the Boltzmann equation [3]. It is also the basis for the construction of the relaxation scheme, a class of simple, efficient TVD schemes for a general system of conservation laws [8] that does not need a Riemann solver nor the local characteristic decomposition.

Despite its simplicity, the system (1.1) possesses the key features of a more general hyperbolic system with relaxation. It thus serves as an ideal model problem to understand the more general ones. This is the reason we, among others, study this model. Our analysis clearly extends to more general systems and asymptotic limits of similar type.

Similar to what was normally done for a kinetic equation [12], we rescale to the long time $t \mapsto t / \varepsilon$ and the weak nonlinearity $f(u) \mapsto \varepsilon f(u)$. Under such a scaling, (1.1) becomes (we still keep the same notation for all variables)

$$
\begin{aligned}
\varepsilon u_{t}^{\varepsilon}+\tilde{v}_{x}^{\varepsilon} & =0, \\
\varepsilon \tilde{v}_{t}^{\varepsilon}+a u_{x}^{\varepsilon} & =-\frac{1}{\varepsilon}\left[\tilde{v}^{\varepsilon}-\varepsilon f\left(u^{\varepsilon}\right)\right] .
\end{aligned}
$$

After introducing $\tilde{v}=\varepsilon v,(1.4)$ becomes

$$
\left(E_{\varepsilon}\right) \quad\left\{\begin{array}{l}
u_{t}^{\varepsilon}+v_{x}^{\varepsilon}=0, \\
\varepsilon^{2} v_{t}^{\varepsilon}+a u_{x}^{\varepsilon}=f\left(u^{\varepsilon}\right)-v^{\varepsilon} .
\end{array}\right.
$$

The initial data associated with $\left(E_{\varepsilon}\right)$ are

$$
\left.\left(u^{\varepsilon}, v^{\varepsilon}\right)\right|_{t=0}=\left(u_{0}^{\varepsilon}, v_{0}^{\varepsilon}\right) \stackrel{x \rightarrow \pm \infty}{\longrightarrow}\left(u_{ \pm}, v_{ \pm}\right), \quad v_{ \pm}=f\left(u_{ \pm}\right) .
$$

Here, for convenience, $v_{ \pm}$is chosen to be the local equilibrium, so there is no initial layer.

Asymptotically it is easy to see that, as $\varepsilon \rightarrow 0,\left(E_{\varepsilon}\right)$ reduces to the following problem

$$
\left(E_{0}\right) \quad\left\{\begin{array}{l}
u_{t}+f(u)_{x}=a u_{x x}, \\
v=f(u)-a u_{x} .
\end{array}\right.
$$

Our goal in this paper is to rigorously justify this asymptotic limit. Since the reduced equation $\left(E_{0}\right)$ is a diffusion equation, we call such a limit the diffusion limit.

Since the reduced equation $\left(E_{0}\right)$ is parabolic, the key smoothness assumption which leads to the above approximation is naturally satisfied. Thus we do not need to impose 
any additional restriction to ensure stability. This can be better understood by using the Chapman-Enskog expansion, which in the case of $\left(E_{\varepsilon}\right)$ reads

$$
v^{\varepsilon}=f\left(u^{\varepsilon}\right)-a u_{x}^{\varepsilon}+\varepsilon^{2} v_{1}^{\varepsilon}+O\left(\varepsilon^{4}\right) .
$$

Applying (1.6) in the system $\left(E_{\varepsilon}\right)$, we obtain, up to an $O\left(\varepsilon^{2}\right)$ error,

$$
v_{1}^{\varepsilon}=f^{\prime}(u)^{2} u_{x}-a\left(f(u)_{x x}+f^{\prime}(u) u_{x x}\right)+a^{2} u_{x x x} .
$$

After dropping all higher-order terms, we get the second-order correction to the limiting form $\left(E_{0}\right)$ :

$$
u_{t}+f(u)_{x}=\left[\left(a-\varepsilon^{2}{f^{\prime}}^{2}\right) u_{x}\right]_{x}+a \varepsilon^{2}\left[f(u)_{x x}+f^{\prime}(u) u_{x x}\right]_{x}-a^{2} \varepsilon^{2} u_{x x x x} .
$$

The equation (1.7) always possesses positive viscosity for $\varepsilon<1$ if the subcharacteristic condition (1.3) is satisfied.

In the present paper, we will investigate the diffusion limit of system $\left(E_{\varepsilon}\right)$, with the initial data prescribed around a traveling wave of $\left(E_{\varepsilon}\right)$. We intend to show that the sequence $\left\{\left(u^{\varepsilon}, v^{\varepsilon}\right)\right\}$ converges, as $\varepsilon \rightarrow 0$, to the unique solution $(u, v)$ of the problem $\left(E_{0}\right)$. Our result shows that, for any solution of the original system $\left(E_{\varepsilon}\right)$, a small perturbation about a traveling wave $\left(U^{\varepsilon}, V^{\varepsilon}\right)$ will persist in the moving frame. Namely, the solution of $\left(E_{\varepsilon}\right)$ will converge to that of the reduced equation $\left(E_{0}\right)$ about its own traveling wave, which is the limit of the $U$-component of the traveling wave of the original system.

In Section 2, we will show that there exist traveling wave solutions with shock profile for $\left(E_{\varepsilon}\right)$, i.e.,

$$
\begin{gathered}
\left(u^{\varepsilon}, v^{\varepsilon}\right)(x, t)=\left(U^{\varepsilon}, V^{\varepsilon}\right)(x-s t) \equiv\left(U^{\varepsilon}, V^{\varepsilon}\right)(z), \\
\left(U^{\varepsilon}, V^{\varepsilon}\right)(z) \rightarrow\left(u_{ \pm}, v_{ \pm}\right) \quad \text { as } \quad z \rightarrow \pm \infty
\end{gathered}
$$

where $\left(u_{-}, u_{+}\right)$is an admissible shock of the corresponding conservation laws. That is, the constants $u_{ \pm}$and $s$ (shock speed) satisfy the Rankine-Hugoniot condition

$$
H\left(u_{+}\right)=0, \quad H(u) \equiv-s\left(u-u_{-}\right)+f(u)-f\left(u_{-}\right)
$$

and the entropy condition [11]

$$
H(u) \begin{cases}<0 & \text { for } u_{+}<u<u_{-}, \\ >0 & \text { for } u_{-}<u<u_{+} .\end{cases}
$$

We also show that, as $\varepsilon \rightarrow 0$, the above traveling wave converges to the traveling wave $\left(U^{0}, V^{0}\right)$ of the reduced equation $\left(E_{0}\right)$ in the $H^{1}$ norm, provided $\int\left(U^{\varepsilon}-U^{0}\right)=0$.

In Section 3, we study the initial-value problem associated with the error (between $\left(u^{\varepsilon}, v^{\varepsilon}\right)$ and $\left.\left(u^{0}, v^{0}\right)\right)$ equation, and we will derive some elementary a priori estimates for the solution which lead to the global wellposedness of the error equation. The energy method employed in this section is similar to that in [13] and [14] where the nonlinear stability of traveling waves to system (1.1) was obtained. The convergence to the local equilibrium is established in Section 4, and the main theorem is proved in Section 5. Furthermore, it will be shown that some higher-order energy estimates hold under additional assumptions on initial data which ensures the compactness.

In the recent literature, the main mathematical tools in justifying the zero relaxation limit are compensated compactness or BV estimates [20]. These techniques, in general, do not work for hyperbolic systems. Therefore, it is interesting to analyze the possibilities of the energy methods for such problems, although in the present 
case the BV estimates still could be obtained under possibly weaker assumptions by monotonicity techniques.

Notations. $H^{m}$ denotes the Sobolev space on $\mathbb{R}$ of order $m$; its norm will be denoted by $\|\cdot\|_{m} . \quad L^{2}\left([0, T] ; H^{k}\right)$ is the space of functions $g(t, x)$ such that $\|g(t, \cdot)\|_{k}$ is in $L^{2}([0, T])$, and similarly for $C^{0}\left([0, T] ; H^{k}\right)$ and $H^{m}\left([0, T] ; H^{k}\right)$. Other norms that will be used occasionally are $\|g\|_{L^{\infty}}=\operatorname{ess} \sup |g|$ and $\left\|\left(g_{1}, g_{2}\right)\right\|_{m}=\left\|g_{1}\right\|_{m}+\left\|g_{2}\right\|_{m}$. Here and elsewhere, $H^{m}$ and $\|\cdot\|_{m}$ refer to the spatial variables only unless otherwise indicated.

The elementary estimates $a b \leq \delta a^{2}+\frac{1}{4 \delta} b^{2}$ for any $\delta>0$, the Schwarz inequality, the Sobolev inequality $\|\cdot\|_{C^{k}} \leq C\|\cdot\|_{m}$ for $m>\frac{1}{2}+k$, will be used freely. Throughout the paper, integrals are taken over $\mathbb{R}$ unless otherwise indicated. $C$ is the generic positive constant independent of $\varepsilon$.

\section{Traveling waves}

To be self-contained, we first state the existence theorem of the traveling wave solution with shock profile for the system $\left(E_{\varepsilon}\right)$. We then will show that it converges, as $\varepsilon \rightarrow 0$, to the traveling wave solution of $\left(E_{0}\right)$ in $H^{1}$. Substituting

$$
(u, v)(x, t)=\left(U^{\varepsilon}, V^{\varepsilon}\right)(z), \quad z=x-s t,
$$

into $\left(E_{\varepsilon}\right)$, we have

$$
\begin{aligned}
-s U_{z}^{\varepsilon}+V_{z}^{\varepsilon} & =0 \\
-s \varepsilon^{2} V_{z}^{\varepsilon}+a U_{z}^{\varepsilon} & =f\left(U^{\varepsilon}\right)-V^{\varepsilon}
\end{aligned}
$$

This also implies

$$
\left(a-\varepsilon^{2} s^{2}\right) U_{z}^{\varepsilon}=f\left(U^{\varepsilon}\right)-V^{\varepsilon} .
$$

Integrating the first equation of $(2.1)$ over $( \pm \infty, z)$, and using the boundary conditions, one has

$$
-s U^{\varepsilon}+V^{\varepsilon}=-s u_{ \pm}+v_{ \pm}=-s u_{ \pm}+f\left(u_{ \pm}\right) .
$$

Combining (2.2) with (2.3) gives

$$
\left(I_{\varepsilon}\right) \quad U_{z}^{\varepsilon}=\frac{H\left(U^{\varepsilon}\right)}{a-\varepsilon^{2} s^{2}} .
$$

Without loss of generality, if we restrict ourselves to the case

$$
u_{+}<u_{-} \quad \text { and } \quad U_{z}^{\varepsilon}<0,
$$

then the ordinary differential equation $\left(I_{\varepsilon}\right)$ with boundary condition $U^{\varepsilon}( \pm \infty)=u_{ \pm}$ admits a unique smooth solution up to a shift provided that $H(U) \rightarrow 0$ at finite order as $U \rightarrow u_{ \pm}$. Moreover, if $f^{\prime}\left(u_{+}\right)<s<f^{\prime}\left(u_{-}\right)$, or $H^{\prime}\left(u_{ \pm}\right) \neq 0$, then $H\left(U^{\varepsilon}\right) \sim$ $-\left|U^{\varepsilon}-u_{ \pm}\right|$as $U^{\varepsilon} \rightarrow u_{ \pm}$. Hence, $\left|\left(U^{\varepsilon}-u_{ \pm}, V^{\varepsilon}-v_{ \pm}\right)(z)\right| \sim \exp \left(-c_{ \pm}|z|\right)$ as $z \rightarrow \pm \infty$ for some constants $c_{ \pm}>0$, independent of $\varepsilon$. If $s=f^{\prime}\left(u_{+}\right)$, or $H^{\prime}\left(u_{+}\right)=0$, then $\left|\left(U^{\varepsilon}-u_{+}, V^{\varepsilon}-v_{+}\right)(z)\right| \sim z^{-1 / k_{+}}$as $z \rightarrow+\infty$, provided $H\left(U^{\varepsilon}\right) \sim-\left|U^{\varepsilon}-u_{+}\right|^{1+k_{+}}$for $k_{+}>0$. We note $k_{+}=n$ if $H^{\prime}\left(u_{+}\right)=\cdots=H^{(n)}\left(u_{+}\right)=0$ and $H^{(n+1)}\left(u_{+}\right) \neq 0$. Thus, we have the existence of traveling wave solutions. 
Lemma 2.1. Assume $H\left(U^{\varepsilon}\right)<0$ for $U^{\varepsilon} \in\left(u_{+}, u_{-}\right)$, and $\left|H\left(U^{\varepsilon}\right)\right| \sim\left|U^{\varepsilon}-u_{ \pm}\right|^{1+k_{ \pm}}$ as $U^{\varepsilon} \rightarrow u_{ \pm}$with $k_{ \pm} \geq 0$. Then there exists a traveling wave solution $\left(U^{\varepsilon}, V^{\varepsilon}\right)(x-s t)$ to $\left(E_{\varepsilon}\right)$ with $\left(U^{\varepsilon}, V^{\varepsilon}\right)( \pm \infty)=\left(u_{ \pm}, v_{ \pm}\right)$, unique up to a shift, and the speed satisfies

$$
\varepsilon^{2} s^{2}<a .
$$

Moreover, as $z \rightarrow \pm \infty$,

$$
\begin{gathered}
\left|\left(U^{\varepsilon}-u_{ \pm}, V^{\varepsilon}-v_{ \pm}\right)(z)\right| \sim \exp \left(-c_{ \pm}|z|\right), \quad \text { if } \quad f^{\prime}\left(u_{+}\right)<s<f^{\prime}\left(u_{-}\right) \\
\left|\left(U^{\varepsilon}-u_{+}, V^{\varepsilon}-v_{+}\right)(z)\right| \sim z^{-\frac{1}{k_{+}}}, \quad \text { if } s=f^{\prime}\left(u_{+}\right) .
\end{gathered}
$$

Remark 1. This existence also includes the case of $\varepsilon=0$; the corresponding wave profile is the solution of the reduced equation.

We now prove that $U^{\varepsilon}$ converges to $U^{0}$ in the $H^{1}$ norm as $\varepsilon \rightarrow 0$ in the case of $f^{\prime}\left(u_{+}\right)<s<f^{\prime}\left(u_{-}\right)$, which will be used in a later section.

Lemma 2.2. Let $U^{\varepsilon}$ and $U^{0}$ be one component of the traveling waves for $\left(E_{\varepsilon}\right)$ and $\left(E_{0}\right)$, respectively, and $\int\left(U^{\varepsilon}-U^{0}\right) d x=0$. Then

$$
\left\|U^{\varepsilon}-U^{0}\right\|_{1} \rightarrow 0 \quad \text { as } \varepsilon \rightarrow 0 \text {. }
$$

Proof. Define $z=x-s t$. Using $\left(E_{\varepsilon}\right)$, we find that $U^{\varepsilon}$ and $U^{0}$ satisfy

$$
\left(I_{\varepsilon}\right) \quad \frac{d U^{\varepsilon}}{d z}=\frac{H\left(U^{\varepsilon}\right)}{a-\varepsilon^{2} s^{2}}
$$

and

$$
\left(I_{0}\right) \quad \frac{d U^{0}}{d z}=\frac{H\left(U^{0}\right)}{a},
$$

respectively. Here the profiles $U^{\varepsilon}$ and $U^{0}$, connecting $u_{+}$to $u_{-}$, are both monotone. The condition $\int\left(U^{\varepsilon}-U^{0}\right) d z=0$ implies that there exists at least one spatial point $z^{*}$, say $z^{*}=0$, such that

$$
U^{\varepsilon}\left(z^{*}\right)=U^{0}\left(z^{*}\right)=\bar{u} \in\left(u_{+}, u_{-}\right) .
$$

Let $z=\left(1-\varepsilon^{2} s^{2} / a\right) \tilde{z}$ and $U^{\varepsilon}(z)=\tilde{U}^{\varepsilon}(\tilde{z})$, then equation $\left(I_{\varepsilon}\right)$ becomes

$$
\frac{d \tilde{U}^{\varepsilon}}{d \tilde{z}}=\frac{H\left(\tilde{U}^{\varepsilon}\right)}{a}
$$

Since $\tilde{U}^{\varepsilon}(\tilde{z}=0)=U^{0}(z=0)=\bar{u}$, one gets

$$
U^{0}(z)=\tilde{U}^{\varepsilon}(z)=U^{\varepsilon}\left(z-\frac{\varepsilon^{2} s^{2}}{a} z\right)
$$

by the uniqueness of the solution to (2.6). Therefore,

$$
\begin{aligned}
U^{\varepsilon}(z)-U^{0}(z) & =U^{\varepsilon}(z)-U^{\varepsilon}\left(z-\frac{\varepsilon^{2} s^{2}}{a} z\right) \\
& =\frac{d U^{\varepsilon}}{d z}\left(z-\theta \frac{\varepsilon^{2} s^{2}}{a} z\right)\left(\frac{\varepsilon^{2} s^{2}}{a} z\right) \quad \text { for } \quad 0<\theta<1 .
\end{aligned}
$$

For some $M>0$ to be determined later, we have

$$
I_{0}=\int_{-M}^{M}\left|U^{\varepsilon}(z)-U^{0}(z)\right|^{2} d z \leq\left(\frac{\varepsilon s}{a}\right)^{4} \max \left|H\left(U^{\varepsilon}\right)\right|^{2} \int_{-M}^{M} z^{2} d z \leq C \varepsilon^{4} M^{3} .
$$


On the other hand,

$$
\begin{aligned}
I_{+} & =\int_{M}^{+\infty}\left|U^{\varepsilon}(z)-U^{0}(z)\right|^{2} d z \\
& \leq 2 \int_{M}^{+\infty}\left|U^{\varepsilon}-u_{+}\right|^{2}+\left|U^{0}(z)-u_{+}\right|^{2} d z \leq C e^{-2 c M}
\end{aligned}
$$

where we have used the fact that the traveling waves tend to $u_{+}$exponentially as $z \rightarrow+\infty$, uniformly in $\varepsilon$, in the case of $f^{\prime}\left(u_{+}\right)<s<f^{\prime}\left(u_{-}\right)$. Hence, for any small positive constant $\delta$, there exists a positive constant $M_{1}$ such that $I_{+}<\frac{\delta}{3}$ for $M \geq M_{1}$. In a similar fashion, we have

$$
I_{-}=\int_{-\infty}^{-M}\left|U^{\varepsilon}(z)-U^{0}(z)\right|^{2} d z \leq \frac{\delta}{3}
$$

for $M \geq M_{2}$. With $M_{0}=\max \left\{M_{1}, M_{2}\right\}$ fixed, letting $\varepsilon$ be suitably small, we obtain $I_{0}<\frac{\delta}{3}$ for $M \geq M_{0}$. Thus,

$$
I=\int\left|U^{\varepsilon}-U^{0}\right|^{2} d z \leq I_{-}+I_{0}+I_{+}<\delta
$$

which yields the convergence in $L^{2}$. The convergence in $H^{1}$ follows immediately by using the equations $\left(I_{\varepsilon}\right)$ and $\left(I_{0}\right)$.

\section{Global existence and uniform estimates}

In this section, we shall establish the global existence for the solutions $\left(u^{\varepsilon}, v^{\varepsilon}\right)$ of the Cauchy problem $\left(E_{\varepsilon}\right)$ with the initial data

$$
u^{\varepsilon}(x, 0)=u_{0}^{\varepsilon}(x), \quad v^{\varepsilon}(x, 0)=v_{0}^{\varepsilon}(x),
$$

for $x \in \mathbb{R}$. Let us consider the following assumptions:

$\left(\mathrm{H}_{1}\right) f$ is a smooth convex function, say $f(u) \in C^{3}(\mathbb{R})$.

$\left(\mathrm{H}_{2}\right)$ The functions $\left(u_{0}^{\varepsilon}, v_{0}^{\varepsilon}\right)$ are uniformly bounded in $L^{\infty}(R)^{2}$. Moreover, the sequence $\left(u_{0}^{\varepsilon}-U^{\varepsilon}, v^{\varepsilon}-V^{\varepsilon}\right)$ converges in $L^{2}(R)^{2}$ to some $\left(u_{0}-U^{0}, v_{0}-V^{0}\right) \in L^{2}(R)^{2}$ as $\varepsilon \rightarrow 0$ where $\left(U^{\varepsilon}, V^{\varepsilon}\right)$ are traveling waves of $\left(E_{\varepsilon}\right)$ discussed in Section 2 .

For the initial data, without loss of generality, we may assume

$$
\int_{-\infty}^{+\infty}\left(u_{0}^{\varepsilon}-U^{\varepsilon}\right)(x) d x=0 .
$$

For some traveling wave $\left(U^{\varepsilon}, V^{\varepsilon}\right)$, given in Section 2, we define

$$
\begin{gathered}
\left(\phi^{\varepsilon}(x, t), \psi^{\varepsilon}(x, t)\right)=\int_{-\infty}^{x}\left(u^{\varepsilon}-U^{\varepsilon}\right)(y) d y,\left(v^{\varepsilon}-V^{\varepsilon}\right)(x), \\
\left(\phi_{0}^{\varepsilon}, \psi_{0}^{\varepsilon}\right)=\int_{-\infty}^{x}\left(u_{0}^{\varepsilon}-U^{\varepsilon}\right)(y) d y,\left(v_{0}^{\varepsilon}-V^{\varepsilon}\right)(x) .
\end{gathered}
$$

Clearly (3.2) implies $\phi_{0}( \pm \infty, t)=0$. Our goal then is to show the existence theorem:

Theorem 3.1. Suppose $\left(\mathrm{H}_{1}\right)$ holds, and $f^{\prime}\left(u_{+}\right)<s<f^{\prime}\left(u_{-}\right)$. Let $\left(U^{\varepsilon}, V^{\varepsilon}\right)(x-s t)$ be a traveling wave solution satisfying (3.2), and assume that $u_{0}^{\varepsilon}-U^{\varepsilon}$ is integrable on 
$\mathbb{R}$ and $\phi_{0}^{\varepsilon} \in H^{3}, \varepsilon \psi_{0}^{\varepsilon} \in H^{2}$. In addition, assume there exist positive constants $\varepsilon_{0}<1$ and $c_{0}$ independent of $\varepsilon$ such that

$$
\begin{gathered}
0<\varepsilon \leq \varepsilon_{0}<1, \quad{f^{\prime}}^{2}<a \text { for } u \in\left(u_{+}, u_{-}\right), \\
\left\|\phi_{0}^{\varepsilon}\right\|_{H^{3}}+\left\|\varepsilon \psi_{0}^{\varepsilon}\right\|_{H^{2}} \leq c_{0} .
\end{gathered}
$$

Then there exists a unique global solution $\left(u^{\varepsilon}, v^{\varepsilon}\right)$ to $\left(E_{\varepsilon}\right)$, with initial condition (1.5), such that

$$
\left(u^{\varepsilon}-U^{\varepsilon}, v^{\varepsilon}-V^{\varepsilon}\right) \in C^{0}\left([0, \infty) ; H^{2}\right) \cap L^{2}\left([0, \infty) ; H^{2}\right)
$$

Moreover,

$$
\begin{gathered}
\left\|\left(u^{\varepsilon}-U^{\varepsilon}, v^{\varepsilon}-V^{\varepsilon}\right)\right\|_{L^{2}\left([0, \infty) ; H^{2}\right)} \leq C, \\
\left\|\left(u^{\varepsilon}-U^{\varepsilon}, \varepsilon\left(v^{\varepsilon}-V^{\varepsilon}\right)\right)\right\|_{C^{0}\left([0, \infty) ; H^{2}\right)} \leq C .
\end{gathered}
$$

To prove Theorem 3.1, we reset the problem $\left(E_{\varepsilon}\right)$ by applying

$$
\left(u^{\varepsilon}, v^{\varepsilon}\right)(x, t)=\left(U^{\varepsilon}, V^{\varepsilon}\right)+\left(\phi_{z}^{\varepsilon}(z, t), \psi^{\varepsilon}(z, t)\right)
$$

where $z=x-s t$ (sometimes we drop the index $\varepsilon$ in the proof for convenience) to get

$$
\begin{aligned}
\left(\phi_{t}-s \phi_{z}\right)+\psi & =0, \\
\varepsilon^{2}\left(\psi_{t}-s \psi_{z}\right)+a \phi_{z z} & =f\left(U^{\varepsilon}+\phi_{z}\right)-f\left(U^{\varepsilon}\right)-\psi .
\end{aligned}
$$

The first equation of (3.5) gives

$$
\psi=-\left(\phi_{t}-s \phi_{z}\right)
$$

Applying (3.6) in the second equation of (3.5), a closed equation for $\phi$ is obtained:

$$
L(\phi)=\varepsilon^{2}\left\{\left(\phi_{t}-s \phi_{z}\right)_{t}-s\left(\phi_{t}-s \phi_{z}\right)_{z}\right\}-a \phi_{z z}+\phi_{t}-s \phi_{z}+f^{\prime}\left(U^{\varepsilon}\right) \phi_{z}=F
$$

where

$$
F=-\left\{f\left(U^{\varepsilon}+\phi_{z}\right)-f\left(U^{\varepsilon}\right)-f^{\prime}\left(U^{\varepsilon}\right) \phi_{z}\right\}=O(1) \phi_{z}^{2}
$$

is a higher order term. The corresponding initial data for (3.7) are

$$
\begin{gathered}
\left.\phi^{\varepsilon}\right|_{t=0}=\phi_{0}^{\varepsilon}(z)=\int_{-\infty}^{z}\left(u_{0}^{\varepsilon}-U^{\varepsilon}\right) d x \\
\left.\phi_{t}^{\varepsilon}\right|_{t=0}=s \phi_{z}^{\varepsilon}-\left.\psi^{\varepsilon}\right|_{t=0}=s \partial_{z} \phi_{0}^{\varepsilon}(z)-\psi_{0}^{\varepsilon} \equiv \phi_{1}^{\varepsilon}(z) .
\end{gathered}
$$

We introduce the solution space of the problem (3.7), (3.9) as

$$
\begin{aligned}
& X(0, T)=\left\{\phi^{\varepsilon}(z, t): \phi \in C^{0}\left([0, T) ; H^{3}\right) \cap C^{1}\left([0, T) ; H^{2}\right),\right. \\
&\left.\left(\phi_{z}, \phi_{t}\right) \in L^{2}\left([0, T) ; H^{2}\right)\right\}, \quad 0<T<+\infty .
\end{aligned}
$$

Clearly, (3.6) implies

$$
\psi \in C^{0}\left([0, T) ; H^{2}\right) \cap L^{2}\left([0, T) ; H^{2}\right) .
$$

Thus, Theorem 3.1 can be obtained from the following proposition. 
Proposition 3.1. Let $N_{0}(t)=\sup _{0 \leq \tau \leq t}\left\{\|\phi(\tau)\|_{3}+\|\varepsilon \psi(\tau)\|_{2}\right\}$. Under the assumptions in Theorem 3.1, the problem (3.7)-(3.9) admits a unique solution $\phi^{\varepsilon} \in X(0,+\infty)$ satisfying

$$
\|\phi(t)\|_{H^{3}(R)}+\|\varepsilon \psi(t)\|_{H^{2}(R)}+\left[\int_{0}^{t}\left\|\left(\psi, \phi_{z}\right)\right\|_{H^{2}}^{2} d \tau\right]^{1 / 2} \leq C N_{0}(0)
$$

where $C$ is a positive constant independent of $\varepsilon$.

Proof of Proposition 3.1. (3.7) can be written as a first-order symmetric hyperbolic system, so the existence of a solution to (3.7)-(3.9) on some time interval $[0, T(\varepsilon)]$ is well known. Furthermore, in order to show that $T(\varepsilon) \geq T$ for some $T$ independent of $\varepsilon$ and that (3.10) holds, it suffices to show that (3.10) holds for $\varepsilon$ sufficiently small under the assumptions that $\phi$ exists on $[0, T]$ and is smooth enough to justify the energy estimates used below.

Next, we will give an a priori estimate for (3.7) with energy estimates. For simplicity in our energy analysis, we assume

$$
f^{\prime \prime}(u) \geq \alpha>0 \quad \text { for } \quad u \in\left(u_{+}, u_{-}\right) .
$$

Methods used later in this section are similar to those in [13, 14]. Multiplying (3.7) by $2 \phi$ yields

$$
2 \phi \cdot L(\phi)=2 F \phi
$$

The left-hand side can be reduced to

$$
\begin{gathered}
\text { L.H.S. }=\left[\phi^{2}+2 \varepsilon^{2} \phi\left(\phi_{t}-s \phi_{z}\right)\right]_{t}-2 \varepsilon^{2}\left(\phi_{t}-s \phi_{z}\right)^{2}+2 a \phi_{z}^{2}-\lambda_{z} \phi^{2}+\{\cdots\}_{z}, \\
\lambda=f^{\prime}\left(U^{\epsilon}\right)-s,
\end{gathered}
$$

where $\{\cdots\}_{z}$ denotes the terms which disappear after integration with respect to $z$ over $\mathbb{R}$. Multiplying (3.7) by $2\left(\phi_{t}-s \phi_{z}\right)$ gives

$$
2\left(\phi_{t}-s \phi_{z}\right) \cdot L(\phi)=2 F\left(\phi_{t}-s \phi_{z}\right) \text {. }
$$

The left hand side is reduced to

$$
\text { L.H.S. }=\left[a \phi_{z}^{2}+\varepsilon^{2}\left(\phi_{t}-s \phi_{z}^{2}\right)\right]_{t}+2\left(\phi_{t}-s \phi_{z}\right)^{2}+2 f^{\prime}(U) \phi_{z}\left(\phi_{t}-s \phi_{z}\right)+\{\cdots\}_{z} .
$$

Hence, the combination of the above equations in the form

$$
\left\{\phi+2\left(\phi_{t}-s \phi_{z}\right)\right\} \cdot L(\phi)=F\left\{\phi+2\left(\phi_{t}-s \phi_{z}\right)\right\}
$$

yields

$$
\begin{aligned}
\left\{E_{1}\left(\phi, \phi_{t}-s \phi_{z}\right)+E_{3}\left(\phi_{z}\right)\right\}_{t}+E_{2}\left(\phi_{z}, \phi_{t}-s \phi_{z}\right)+E_{4}(\phi)+\{\cdots\}_{z} & \\
& =F\left\{\phi+2\left(\phi_{t}-s \phi_{z}\right)\right\}
\end{aligned}
$$

where

$$
\begin{gathered}
E_{1}\left(\phi, \phi_{t}-s \phi_{z}\right)=\varepsilon^{2}\left(\phi_{t}-s \phi_{z}\right)^{2}+\varepsilon^{2} \phi\left(\phi_{t}-s \phi_{z}\right)+\frac{1}{2} \phi^{2} \\
E_{2}\left(\phi_{z}, \phi_{t}-s \phi_{z}\right)=\left(2-\varepsilon^{2}\right)\left(\phi_{t}-s \phi_{z}\right)^{2}+2 f^{\prime}\left(U^{\varepsilon}\right) \phi_{z}\left(\phi_{t}-s \phi_{z}\right)+a \phi_{z}^{2}, \\
E_{3}\left(\phi_{z}\right)=a \phi_{z}^{2}, \quad E_{4}(\phi)=-\lambda_{z}\left(\frac{\phi^{2}}{2}\right) .
\end{gathered}
$$


Since $\lambda_{z}=f^{\prime \prime}\left(U^{\varepsilon}\right) U_{z}^{\varepsilon}$ and $U_{z}^{\varepsilon}<0, f^{\prime \prime}\left(U^{\varepsilon}\right) \geq \alpha$, one gets

$$
E_{4}(\phi) \geq \frac{\alpha}{2}\left|U_{z}^{\varepsilon}\right| \phi^{2}
$$

It is easy to see that (3.3) ensures the positive definiteness of the energies $E_{1}$ and $E_{2}$. Therefore,

$$
\begin{gathered}
c\left\{\phi^{2}+\epsilon^{2}\left(\phi_{t}-s \phi_{z}\right)^{2}\right\} \leq E_{1} \leq C\left\{\phi^{2}+\epsilon^{2}\left(\phi_{t}-s \phi_{z}\right)^{2}\right\} \\
E_{2} \geq c\left\{\phi_{z}^{2}+\left(\phi_{t}-s \phi_{z}\right)^{2}\right\} .
\end{gathered}
$$

In summary, we have:

Lemma 3.1. If (3.3) holds, then

$$
\begin{gathered}
\|\phi(t)\|_{1}^{2}+\varepsilon^{2}\|\psi(t)\|^{2}+\int_{0}^{t}\left\|\left(\psi, \phi_{z}\right)(\tau)\right\|^{2} d \tau+\int_{0}^{t} \int_{R}\left|U_{z}^{\varepsilon}\right| \phi^{2} d z d \tau \\
\leq C\left\{\left\|\phi_{0}\right\|_{1}^{2}+\varepsilon^{2}\left\|\psi_{0}\right\|^{2}+\int_{0}^{t} \int_{R}|F|(|\phi|+|\psi|) d z d \tau\right\}
\end{gathered}
$$

for $t \in[0, T]$.

The next step is to obtain higher order energy estimates in a manner similar to the preceding arguments. Taking a spatial derivative $\partial_{z}$ over (3.7) and defining $\partial_{z} \phi=\Phi$, one obtains

$$
\partial_{z} L(\phi)=L^{\varepsilon}\left(\phi_{z}\right)+\lambda_{z} \phi_{z}=L(\Phi)+\lambda_{z} \Phi
$$

Rearranging

$$
\left\{\phi_{z}+2\left(\phi_{t}-s \phi_{z}\right)_{z}\right\} \cdot \partial_{z} L(\phi)=2 F_{z}\left\{\phi_{z}+2\left(\phi_{t}-s \phi_{z}\right)_{z}\right\},
$$

by a similar argument as in the proof of Lemma 3.1, one gets

$$
\begin{aligned}
& \left\{\varepsilon^{2}\left(\Phi_{t}-s \Phi_{z}\right)^{2}+\varepsilon^{2} \Phi\left(\Phi_{t}-s \Phi_{z}\right)+\frac{1}{2} \Phi^{2}+a \Phi_{z}^{2}\right\}_{t}+\tilde{E}+G+\{\cdots\}_{z} \\
& \quad=F_{z}\left\{\Phi+2\left(\Phi_{t}-s \Phi_{z}\right)\right\}
\end{aligned}
$$

where

$$
\begin{aligned}
& \tilde{E}=\left(2-\varepsilon^{2}\right)\left(\Phi_{t}-s \Phi_{z}\right)^{2}+2 f^{\prime}\left(U^{\varepsilon}\right) \Phi_{z}\left(\Phi_{t}-s \Phi_{z}\right)+a \Phi_{z}^{2}, \\
& G=2 \lambda_{z} \Phi\left(\Phi_{t}-s \Phi_{z}\right)+\frac{\lambda_{z}}{2} \Phi^{2} .
\end{aligned}
$$

Under condition (3.3), one has

$$
\tilde{E} \geq c\left\{\Phi_{z}^{2}+\left(\Phi_{t}-s \Phi_{z}\right)^{2}\right\}
$$

After the integration with respect to $t$ and $z$, (3.16) gives

$$
\begin{gathered}
\|\Phi(t)\|_{1}^{2}+\varepsilon^{2}\left\|\left(\Phi_{t}-s \Phi_{z}\right)\right\|^{2}+\int_{0}^{t}\left\|\left(\Phi_{t}-s \Phi_{z}, \Phi_{z}\right)(\tau)\right\|^{2} d \tau \\
\leq C\left\{\left\|\Phi_{0}\right\|_{1}^{2}+\varepsilon^{2}\left\|\left(\Phi_{t}-s \Phi_{z}\right)(0)\right\|^{2}+\int_{0}^{t} \int|G| d z d \tau\right. \\
\left.+\int_{0}^{t} \int_{R}\left|F_{z}\right|\left(|\Phi|+\left|\left(\Phi_{t}-s \Phi_{z}\right)\right|\right) d z d \tau\right\}
\end{gathered}
$$


where $\Phi_{0}=\phi_{0}^{\prime}$. Using the estimate in Lemma 3.1,

$$
\begin{aligned}
\int_{0}^{t} \int_{R}|G| d z d \tau \leq & \int_{0}^{t} \int_{R}\left|U_{z}^{\varepsilon}\right|\left[\left|\Phi\left(\Phi_{t}-s \Phi_{z}\right)\right|+\Phi^{2}\right] d z d \tau \\
\leq & \frac{1}{2} \int_{0}^{t} \int_{R}\left|\Phi_{t}-s \Phi_{z}, \Phi\right|^{2} d z d \tau+C \int_{0}^{t} \int_{R}|\Phi|^{2} d z d \tau \\
\leq & \frac{1}{2} \int_{0}^{t} \int_{R}\left|\Phi_{t}-s \Phi_{z}, \Phi\right|^{2} d z d \tau \\
& +C\left\{\left\|\phi_{0}\right\|_{1}^{2}+\varepsilon^{2}\left\|\psi_{0}\right\|^{2}+\int_{0}^{t} \int_{R}|F|(|\phi|+|\psi|) d z d \tau\right\}
\end{aligned}
$$

where we have used the boundedness of $\left|\lambda_{z}\right|$.

Substituting (3.18) into (3.17), and replacing $\Phi$ by $\partial_{z} \phi$, we have

Lemma 3.2. If (3.3) holds, then

$$
\begin{array}{r}
\left\|\partial_{z} \phi\right\|_{1}^{2}+\varepsilon^{2}\left\|\partial_{z} \psi\right\|^{2}+\int_{0}^{t}\left\|\left(\partial_{z} \psi, \partial_{z} \phi_{z}\right)\right\|^{2} d \tau \leq C\left\{\left\|\phi_{0}\right\|_{2}^{2}+\varepsilon^{2}\left\|\psi_{0}\right\|_{1}^{2}\right. \\
\left.+\int_{0}^{t} \int_{R}|F|(|\phi|+|\psi|) d z d \tau+\int_{0}^{t} \int_{R}\left|F_{z}\right|\left(\left|\partial_{z} \phi\right|+\left|\partial_{z} \psi\right|\right) d z d \tau\right\}
\end{array}
$$

for $t \in[0, T]$.

Finally, we consider the equality

$$
\partial_{z}^{2} \phi \cdot \partial_{z}^{2} L(\phi)+2 \partial_{z}^{2}\left(\phi_{t}-s \phi_{z}\right) \cdot \partial_{z}^{2} L(\phi)=\partial_{z}^{2} F\left\{\partial_{z}^{2} \phi+2 \partial_{z}^{2}\left(\phi_{t}-s \phi_{z}\right)\right\}
$$

After some manipulations, we get the following for $\Psi=\partial_{z}^{2} \phi$,

$$
\begin{aligned}
{\left[\varepsilon ^ { 2 } \left(\Psi_{t}\right.\right.} & \left.\left.-s \Psi_{z}\right)^{2}+a \Psi_{z}^{2}+\varepsilon^{2} \Psi\left(\Psi_{t}-s \Psi_{z}\right)+\frac{1}{2} \Psi^{2}\right]_{t}+\left(2-\varepsilon^{2}\right)\left(\Psi_{t}-s \Psi_{z}\right)^{2} \\
& +2 f^{\prime}\left(U^{\varepsilon}\right) \Psi_{z}\left(\Psi_{t}-s \Psi_{z}\right)+a \Psi_{z}^{2}+4 \lambda_{z} \Psi\left(\Psi_{t}-s \Psi_{z}\right) \\
& +\frac{3}{2} \lambda_{z} \Psi^{2}+\lambda_{z z} \Psi \phi_{z}+2 \lambda_{z z} \phi_{z}\left(\Psi_{t}-s \Psi_{z}\right)+\{\cdots\}_{z} \\
=F_{z z} & {\left[\Psi+2\left(\Psi_{t}-s \Psi_{z}\right)\right] . }
\end{aligned}
$$

Integrating over $[0, t] \times \mathbb{R}$, using the fact that $\lambda_{z}, \lambda_{z z}$ are smooth bounded functions, and applying Young's inequality to $4 \lambda_{z} \Psi\left(\Psi_{t}-s \Psi_{z}\right)$ and $2 \lambda_{z z} \phi_{z}\left(\Psi_{t}-s \Psi_{z}\right)$, we get the following estimate on $\Psi=\partial_{z}^{2} \phi$.

Lemma 3.3. If (3.3) holds, then

$$
\begin{gathered}
\left\|\partial_{z}^{2} \phi(t)\right\|_{1}^{2}+\varepsilon^{2}\left\|\partial_{z}^{2} \psi\right\|^{2}+\frac{1}{3} \int_{0}^{t}\left\|\left(\partial_{z}^{2} \psi, \partial_{z}^{2} \phi_{z}\right)\right\|^{2} d \tau-C \int_{0}^{t}\left\{\left\|\partial_{z}^{2} \phi\right\|^{2}+\left\|\phi_{z}\right\|^{2}\right\} d \tau \\
\leq C\left\{\left\|\partial_{z}^{2} \phi(0)\right\|_{1}^{2}+\varepsilon^{2}\left\|\partial_{z}^{2} \psi(0)\right\|^{2}+\int_{0}^{t} \int_{R}\left|F_{z z}\right|\left(\left|\partial_{z}^{2} \phi\right|+\left|\partial_{z}^{2} \psi\right|\right) d z d \tau\right\}
\end{gathered}
$$


Successively combining the estimates in Lemmas 3.1-3.3, we have

$$
\begin{aligned}
\|\phi(t)\|_{3}^{2}+\varepsilon^{2}\|\psi(t)\|_{2}^{2} & +\int_{0}^{t} \int\left|U_{z}^{\varepsilon}\right| \phi^{2} d z d \tau+\int_{0}^{t}\left\|\left(\psi, \phi_{z}\right)\right\|_{2}^{2} d \tau \\
\leq C\left\{\|\phi(0)\|_{3}^{2}\right. & +\varepsilon^{2}\left\|\psi_{0}\right\|_{2}^{2}+\int_{0}^{t} \int_{R}\left\{|F|(|\phi|+|\psi|)+\left|F_{z}\right|\left(\left|\partial_{z} \phi\right|+\left|\partial_{z} \psi\right|\right)\right. \\
& \left.\left.+\left|F_{z z}\right|\left(\left|\partial_{z}^{2} \phi\right|+\left|\partial_{z}^{2} \psi\right|\right)\right\} d z d \tau\right\} .
\end{aligned}
$$

By recalling the definition of $F$ in (3.8), we have

$$
\begin{aligned}
|F| & =O(1)\left|\phi_{z}\right|^{2}, \\
\left|F_{z}\right| & =O(1)\left(\left|\phi_{z}\right|^{2}+\left|\phi_{z}\right|\left|\phi_{z z}\right|\right), \\
\left|F_{z z}\right| & =O(1)\left(\left|\phi_{z}\right|^{2}+\left|\phi_{z z}^{2}\right|+\left|\phi_{z} \phi_{z z z}\right|\right) .
\end{aligned}
$$

Since $\sup _{z}\left\{|\phi|,\left|\phi_{z}\right|,\left|\phi_{z z}\right|\right\} \leq C N_{0}(t)$, the integral on the right-hand side of (3.22) is dominated by

$$
C N_{0}(t) \int_{0}^{t}\left\|\left(\psi, \phi_{z}\right)\right\|_{2}^{2} d \tau
$$

Hence,

$$
N_{0}^{2}(t)+\left(1-C N_{0}(t)\right) \int_{0}^{t}\left\|\left(\psi, \phi_{z}\right)\right\|_{2}^{2} d \tau+\int_{0}^{t} \int_{R}\left|U_{z}^{\varepsilon}\right| \phi^{2} d z d \tau \leq C N_{0}^{2}(0) .
$$

Therefore, assuming $N_{0}(T)<\frac{1}{2 C}$, we obtain the desired estimate

$$
N_{0}^{2}(t)+\int_{0}^{t}\left\|\left(\psi, \phi_{z}\right)\right\|_{2}^{2} d \tau+\int_{0}^{t}\left\|\sqrt{\left|U_{z}^{\varepsilon}\right|} \phi\right\|_{2}^{2} d \tau \leq C N_{0}^{2}(0)
$$

for $t \in[0, T]$. This estimate combined with local existence yields Proposition 3.1.

\section{Convergence to the local equilibrium}

A necessary step to establish the limit is to study the convergence to the local equilibrium $v=f(u)-a u_{x}$. Additional a priori estimates for $\phi_{t}^{\varepsilon}$ and the equilibrium will assure this.

To this end, we multiply $\partial_{t} \cdot L(\phi)$ by $2 \phi_{t}$ and $2\left(\phi_{t}-s \phi_{z}\right)_{t}$, respectively. Noting that $\partial_{t} L(\phi)=L\left(\phi_{t}\right)=L(\tilde{\phi})$, similar arguments as in Lemma 3.1 yield the estimates for $\phi_{t}$.

Lemma 4.1. There exists a positive constant $C$ such that if (3.3) holds, then

$$
\begin{gathered}
\left\|\phi_{t}(t)\right\|_{1}^{2}+\left\|\varepsilon \psi_{t}(t)\right\|^{2}+\int_{0}^{t}\left\|\left(\psi_{\tau}, \partial_{z} \phi_{\tau}\right)(\tau)\right\|^{2} d \tau+\int_{0}^{t} \int\left|U_{z}^{\varepsilon}\right| \phi_{\tau}^{2} d z d \tau \\
\leq C\left\{\left\|\phi_{t}(0)\right\|_{1}^{2}+\left\|\varepsilon \psi_{t}(0)\right\|^{2}+\int_{0}^{t} \int_{R}\left|F_{\tau}\right|\left(\left|\phi_{\tau}\right|+\left|\psi_{\tau}\right|\right) d z d \tau\right\}
\end{gathered}
$$

for $t \in[0, T]$.

By the definition of $F$ in (3.8),

$$
\left|F_{\tau}\right|=O(1)\left|\phi_{z}\right|\left|\phi_{z \tau}\right| \text {. }
$$


The integral on the right-hand side of (4.1) is bounded by

$$
\begin{aligned}
C \int_{0}^{t} & \int_{R}\left|\phi_{z} \phi_{z \tau}\right|\left(\left|\partial_{\tau} \phi\right|+\left|\partial_{\tau} \psi\right|\right) d z d \tau \\
& \leq C N_{0}(t) \int_{0}^{t} \int_{R}\left\{\left|\phi_{z \tau}\right|^{2}+\left|\phi_{\tau}\right|^{2}+\left|\psi_{\tau}\right|^{2}\right\} d z d \tau \\
& \leq C N_{0}(t)\left\{\int_{0}^{t}\left\|\left(\psi_{\tau}, \partial_{z} \phi_{\tau}\right)\right\|^{2} d \tau+\int_{0}^{t}\left\|\left(\psi, \phi_{z}\right)\right\|^{2} d \tau\right\}
\end{aligned}
$$

where we have used the Sobolev inequality and (3.6). Assuming $C N_{0}(t)<\frac{1}{3}$ for $0 \leq t \leq T$, applying (4.2) to (4.1) yields

$$
\begin{gathered}
\left\|\phi_{t}\right\|_{1}^{2}+\left\|\varepsilon \psi_{t}\right\|^{2}+\frac{2}{3} \int_{0}^{t}\left\|\left(\psi_{\tau}, \partial_{z} \phi_{\tau}\right)\right\|^{2} d \tau+\int_{0}^{t} \int_{R}\left|U_{z}^{\varepsilon}\right| \phi_{\tau}^{2} d z d \tau \\
\leq C\left\{\left\|\phi_{t}(0)\right\|_{1}^{2}+\left\|\varepsilon \psi_{t}(0)\right\|^{2}\right\}+\frac{1}{3} \int_{0}^{t}\left\|\left(\psi, \phi_{z}\right)\right\|^{2} d \tau
\end{gathered}
$$

Next, we estimate the higher order of $\phi_{t}$. We begin with the equality,

$$
\left\{\phi_{z t}+2\left(\phi_{t}-s \phi_{z}\right)_{z t}\right\} \cdot \partial_{z t} L(\phi)=F_{z t}\left\{\phi_{z t}+2\left(\phi_{t}-s \phi_{z}\right)_{z t}\right\} \text {. }
$$

Note that $\partial_{z t} L(\phi)=L\left(\phi_{z t}\right)+f^{\prime}\left(U^{\varepsilon}\right)_{z} \phi_{z t}=L(\tilde{\psi})+\lambda_{z} \tilde{\psi}$ with $\tilde{\psi}=\phi_{z t}$. By a similar argument, we have (compare with (3.17))

$$
\begin{gathered}
\|\tilde{\psi}\|_{1}^{2}+\left\|\varepsilon\left(\tilde{\psi}_{t}-s \tilde{\psi}_{z}\right)\right\|^{2}+\int_{0}^{t}\left\|\left(\tilde{\psi}_{\tau}-s \tilde{\psi}_{z}, \partial_{z} \tilde{\psi}\right)(\tau)\right\|^{2} d \tau \\
\leq C\left\{\|\tilde{\psi}(0)\|_{1}^{2}+\left\|\varepsilon\left(\tilde{\psi}_{t}-s \tilde{\psi}_{z}\right)(0)\right\|^{2}+\int_{0}^{t} \int|\tilde{G}| d z d \tau\right. \\
\left.+\int_{0}^{t} \int_{R}\left|F_{z \tau}\right|\left(|\tilde{\psi}|+\left|\tilde{\psi}_{\tau}-s \tilde{\psi}_{z}\right|\right) d z d \tau\right\}
\end{gathered}
$$

where $\tilde{G}=2 \lambda_{z} \tilde{\psi}\left(\tilde{\psi}_{t}-s \tilde{\psi}_{z}\right)+\frac{1}{2} \lambda_{z} \tilde{\psi}^{2}$. That is,

$$
\begin{gathered}
\left\|\phi_{t z}\right\|_{1}^{2}+\left\|\varepsilon \psi_{t z}\right\|^{2}+\int_{0}^{t}\left\|\partial_{z \tau} \psi, \partial_{z \tau} \phi_{z}\right\|^{2} d \tau \\
\leq C\left\{\|\tilde{\psi}(0)\|_{1}^{2}+\left\|\varepsilon\left(\tilde{\psi}_{t}-s \tilde{\psi}_{z}\right)(0)\right\|^{2}+\int_{0}^{t} \int|\tilde{G}| d z d \tau\right. \\
\left.+\int_{0}^{t} \int_{R}\left|F_{z \tau}\right|\left(|\tilde{\psi}|+\left|\tilde{\psi}_{\tau}-s \tilde{\psi}_{z}\right|\right) d z d \tau\right\}
\end{gathered}
$$

Clearly,

$$
\begin{aligned}
C \int_{0}^{t} \int_{R}|\tilde{G}| d z d \tau & \leq \frac{1}{3} \int_{0}^{t} \int_{R}\left|\left(\tilde{\psi}_{\tau}-s \tilde{\psi}_{z}, \tilde{\psi}_{z}\right)\right|^{2} d z d \tau+C \int_{0}^{t} \int_{R}|\tilde{\psi}|^{2} d z d \tau \\
& \leq \frac{1}{3} \int_{0}^{t}\left\|\partial_{z \tau} \psi, \partial_{z \tau} \phi_{z}\right\|^{2} d \tau+C \int_{0}^{t}\left\|\left(\psi_{\tau}, \partial_{z} \phi_{\tau}\right)\right\|^{2} d \tau
\end{aligned}
$$

where we have used the boundedness of $\lambda_{z}$. Considering

$$
\begin{aligned}
\left|F_{z t}\right| & =O(1)\left\{\left|\phi_{z} \phi_{z t}\right|+\left|\phi_{z z} \phi_{z t}\right|+\left|\phi_{z} \phi_{z z t}\right|\right\} \\
& =O(1)\left\{\left|\phi_{z} \tilde{\psi}\right|+\left|\phi_{z z} \tilde{\psi}\right|+\left|\phi_{z} \tilde{\psi}_{z}\right|\right\},
\end{aligned}
$$


the last integral in (4.4) is dominated by

$$
\begin{aligned}
C N_{0}( & (t) \int_{0}^{t} \int_{R}\left\{\tilde{\psi}^{2}+\tilde{\psi}_{z}^{2}+\left(\tilde{\psi}_{\tau}-s \tilde{\psi}_{z}\right)^{2}\right\} d z d \tau \\
\leq & \frac{1}{3} \int_{0}^{t}\left\|\left(\partial_{z} \psi_{\tau}, \partial_{z}^{2} \phi_{\tau}\right)\right\|^{2} d \tau+\frac{1}{3} \int_{0}^{t}\left\|\left(\psi_{\tau}, \partial_{z} \phi_{\tau}\right)\right\|^{2} d \tau
\end{aligned}
$$

By substituting (4.5)-(4.6) into (4.4) with $\tilde{\psi}$ replaced by $\phi_{t z}$, we have

$$
\begin{aligned}
& \left\|\partial_{z} \phi_{t}\right\|_{1}^{2}+\left\|\varepsilon \partial_{z} \psi_{t}\right\|^{2}+\frac{1}{3} \int_{0}^{t}\left\|\left(\partial_{z} \psi_{\tau}, \partial_{z}^{2} \phi_{\tau}\right)\right\|^{2} d \tau \\
& \leq C\left\{\left\|\partial_{z} \phi_{t}(0)\right\|_{1}^{2}+\left\|\varepsilon \partial_{z} \psi_{t}(0)\right\|^{2}+\int_{0}^{t}\left\|\left(\psi_{\tau}, \partial_{z} \phi_{\tau}\right)\right\|^{2} d \tau\right\} .
\end{aligned}
$$

Using (4.3) and (3.23), the last integral in the above inequality is dominated by

$$
\begin{gathered}
C\left\{\left\|\phi_{t}(0)\right\|_{1}^{2}+\left\|\varepsilon \psi_{t}(0)\right\|^{2}+\int_{0}^{t}\left\|\left(\psi, \partial_{z} \phi\right)\right\|^{2} d \tau\right\} \\
\leq C\left\{\left\|\phi_{t}(0)\right\|_{1}^{2}+\left\|\varepsilon \psi_{t}(0)\right\|^{2}+N_{0}^{2}(0)\right\} .
\end{gathered}
$$

Combining these estimates with (4.3) yields

$$
\begin{gathered}
\left\|\phi_{t}\right\|_{2}^{2}+\left\|\varepsilon \psi_{t}\right\|_{1}^{2}+\int_{0}^{t}\left\|\left(\psi_{\tau}, \partial_{z} \phi_{\tau}\right)\right\|_{1}^{2} d \tau+\int_{0}^{t} \int_{R}\left|U_{z}^{\varepsilon}\right| \phi_{\tau}^{2} d z d \tau \\
\leq C\left\{\left\|\phi_{t}(0)\right\|_{2}^{2}+\left\|\varepsilon \psi_{t}(0)\right\|_{1}^{2}+N_{0}^{2}(0)\right\}
\end{gathered}
$$

Using the identity

$$
\partial_{t} \psi=\partial_{t} \psi-s \partial_{z} \psi+s \partial_{z} \psi
$$

we have

$$
\begin{aligned}
\left\|\varepsilon \partial_{t} \psi\right\|_{1}^{2} & \geq\left\|\varepsilon\left(\partial_{t} \psi-s \partial_{z} \psi\right)\right\|_{1}^{2}-\varepsilon^{2} s^{2}\left\|\psi_{z}\right\|_{1}^{2} \\
& \geq\left\|\varepsilon\left(\partial_{t} \psi-s \partial_{z} \psi\right)\right\|_{1}^{2}-\varepsilon^{2} s^{2}\|\psi\|_{2}^{2}
\end{aligned}
$$

Now (3.23) yields

$$
\varepsilon^{2} s^{2}\|\psi\|_{2}^{2}=s^{2}\|\varepsilon \psi\|_{2}^{2} \leq C s^{2} N_{0}^{2}(0)
$$

and

$$
\begin{aligned}
\int_{0}^{t}\left\|\partial_{\tau} \psi\right\|_{1}^{2} & \geq \int_{0}^{t}\left\|\left(\partial_{t} \psi-s \partial_{z} \psi\right)\right\|_{1}^{2} d \tau-s^{2} \int_{0}^{t}\left\|\psi_{z}\right\|_{1}^{2} d \tau \\
& \geq \int_{0}^{t}\left\|\left(\partial_{\tau} \psi-s \partial_{z} \psi\right)\right\|_{1}^{2}-C s^{2} N_{0}^{2}
\end{aligned}
$$

Applying a similar argument to the term $\left\|\varepsilon \partial_{t} \psi(0)\right\|_{1}^{2},(4.7)$ yields

$$
\begin{gathered}
\left\|\phi_{t}\right\|_{2}^{2}+\left\|\varepsilon\left(\psi_{t}-s \psi_{z}\right)\right\|_{1}^{2}+\frac{1}{2} \int_{0}^{t}\left\|\left(\psi_{\tau}, \partial_{z} \phi_{\tau}\right)\right\|_{1}^{2} d \tau+\frac{1}{2} \int_{0}^{t}\left\|\psi_{\tau}-s \psi_{z}\right\|_{1}^{2} d \tau \\
\leq C\left\{\left\|\phi_{t}(0)\right\|_{2}^{2}+\left\|\varepsilon\left(\partial_{t} \psi-s \psi_{z}\right)(0)\right\|_{1}^{2}+N_{0}^{2}(0)\right\}
\end{gathered}
$$


Adding this estimate to (3.23), we get

$$
\begin{aligned}
& N_{0}^{2}(t)+\left\|\phi_{t}(t)\right\|_{2}^{2}+\left\|\varepsilon\left(\psi_{t}-s \psi_{z}\right)\right\|_{1}^{2}+\int_{0}^{t}\left\|\left(\psi, \partial_{z} \phi\right)\right\|_{2}^{2} d \tau \\
&+\int_{0}^{t}\left\|\partial_{\tau}\left(\psi, \partial_{z} \phi\right)\right\|_{1}^{2} d \tau+\int_{0}^{t}\left\|\left(\psi_{\tau}-s \psi_{z}\right)\right\|_{1}^{2} d \tau \\
& \leq C\left\{N_{0}^{2}(0)+\left\|\phi_{t}(0)\right\|_{2}^{2}+\left\|\varepsilon\left(\psi_{t}-s \psi_{z}\right)(0)\right\|_{1}^{2}\right\}
\end{aligned}
$$

Defining

$$
\begin{aligned}
N(t)= & N_{0}^{2}(t)+\sup _{0 \leq \tau \leq t}\left\{\left\|\phi_{\tau}(\tau)\right\|_{2}^{2}+\left\|\varepsilon\left(\psi_{\tau}-s \psi_{z}\right)(\tau)\right\|^{2}\right\} \\
M(t)= & \int_{0}^{t}\left\|\left(\psi, \partial_{z} \phi\right)\right\|_{2}^{2} d \tau+\int_{0}^{t}\left\|\partial_{\tau}\left(\psi, \partial_{z} \phi\right)\right\|_{1}^{2} d \tau \\
& \quad+\int_{0}^{t}\left\|\left(\psi_{\tau}-s \psi_{z}\right)\right\|_{1}^{2} d \tau
\end{aligned}
$$

we obtain, from (4.8), that there exists $\varepsilon_{0}>0$ such that, when $0<\varepsilon<\varepsilon_{0}$,

$$
N(t)+M(t) \leq C D_{0}
$$

where, by (3.5),

$$
\begin{aligned}
D_{0}=\| & \phi_{0}^{\varepsilon}\left\|_{3}^{2}+\right\| \varepsilon \psi^{\varepsilon}(0)\left\|_{2}^{2}+\right\| \phi_{t}^{\varepsilon}(0) \|_{2}^{2} \\
& +\frac{1}{\varepsilon}\left\|\left(\psi^{\varepsilon}-f\left(U^{\varepsilon}+\phi_{z}^{\varepsilon}\right)+f\left(U^{\varepsilon}\right)+a \phi_{z z}^{\varepsilon}\right)(z, 0)\right\|_{1}^{2} .
\end{aligned}
$$

It is clear that if initially we assume

$$
\left\|\phi_{0}^{\varepsilon}\right\|_{3}+\varepsilon\left\|\psi_{0}^{\varepsilon}\right\|_{2}+\left\|\phi_{t}^{\varepsilon}(0)\right\|_{2} \leq c_{0}
$$

and

$$
\frac{1}{\varepsilon}\left\|\left(\psi^{\varepsilon}-f\left(U^{\varepsilon}+\phi_{z}^{\varepsilon}\right)+f\left(U^{\varepsilon}\right)+a \phi_{z z}^{\varepsilon}\right)(z, 0)\right\|_{H^{1}(\mathbb{R})} \leq c_{0}
$$

then the above estimates lead to

$$
\left\|\left(\psi^{\varepsilon}-f\left(U^{\varepsilon}+\phi_{z}^{\varepsilon}\right)+f\left(U^{\varepsilon}\right)+a \phi_{z z}^{\varepsilon}\right)(t)\right\|_{L^{\infty}\left(\mathbb{R}_{+} ; L^{2}(\mathbb{R})\right)} \leq C \varepsilon
$$

and

$$
\left\|\psi^{\varepsilon}-f\left(U^{\varepsilon}+\phi_{z}^{\varepsilon}\right)+f\left(U^{\varepsilon}\right)+a \phi_{z z}^{\varepsilon}\right\|_{H^{1}\left(\mathbb{R} \times \mathbb{R}_{+}\right)} \leq C \varepsilon^{2} .
$$

In conclusion, we have proved

Theorem 4.1. Assume, in addition to the hypotheses of Theorem 3.1, that

$$
\left\|\phi_{t}^{\varepsilon}(0)\right\|_{2} \leq c_{0}
$$

and

$$
\frac{1}{\varepsilon}\left\|\left(\psi^{\varepsilon}-f\left(U^{\varepsilon}+\phi_{z}^{\varepsilon}\right)+f\left(U^{\varepsilon}\right)+a \phi_{z z}^{\varepsilon}\right)(z, 0)\right\|_{1} \leq c_{0},
$$

then the global solution $\phi^{\varepsilon}$ for the Cauchy problem (3.7), (3.9) satisfies

$\sup _{0 \leq t \leq T}\left\{\|\phi(t)\|_{3}^{2}+\|\varepsilon \psi(t)\|_{2}^{2}+\left\|\phi_{t}(t)\right\|_{2}^{2}\right\}+\int_{0}^{T}\left\|\left(\psi, \phi_{z}\right)\right\|_{2}^{2} d \tau+\int_{0}^{T}\left\|\partial_{\tau}\left(\psi, \phi_{z}\right)\right\|_{1}^{2} d \tau \leq C$ 
for any $T \geq 0$, and

$$
\begin{gathered}
\left\|\psi^{\varepsilon}-f\left(U^{\varepsilon}+\phi_{z}^{\varepsilon}\right)+f\left(U^{\varepsilon}\right)+a \phi_{z z}^{\varepsilon}\right\|_{H^{1}\left(\mathbb{R} \times \mathbb{R}_{+}\right)} \leq C \varepsilon^{2} \\
\sup _{0 \leq t \leq T}\left\|\left(\psi^{\varepsilon}-f\left(U^{\varepsilon}+\phi_{z}^{\varepsilon}\right)+f\left(U^{\varepsilon}\right)+a \phi_{z z}^{\varepsilon}\right)(t)\right\|_{L^{2}(\mathbb{R})} \leq C \varepsilon .
\end{gathered}
$$

Consequently, we have the desired solution of problem $\left(E_{\varepsilon}\right),(1.5)$, through the relation (3.4).

Theorem 4.2. Assume, in addition to the hypothesis of Theorem 3.1, that

$$
\|\left(v_{0}^{\varepsilon}-V^{\varepsilon}-s\left(u_{0}^{\varepsilon}-U^{\varepsilon}\right) \|_{2} \leq c_{0}\right.
$$

and

$$
\frac{1}{\varepsilon}\left\|v_{0}^{\varepsilon}-f\left(u_{0}^{\varepsilon}\right)+a \partial_{x} u_{0}^{\varepsilon}\right\|_{1} \leq c_{0}
$$

then the global solution $\left(u^{\varepsilon}, v^{\varepsilon}\right)$ for the Cauchy problem $\left(E_{\varepsilon}\right),(1.5)$ satisfies, for any $T \geq 0$,

and

$$
\begin{gathered}
\sup _{0 \leq t \leq T}\left\|\left(u^{\varepsilon}-U^{\varepsilon}, \varepsilon\left(v^{\varepsilon}-V^{\varepsilon}\right)\right)\right\|_{2}^{2}+\int_{0}^{T}\left\|\left(u^{\varepsilon}-U^{\varepsilon}, v^{\varepsilon}-V^{\varepsilon}\right)\right\|_{2}^{2} d \tau \leq C, \\
\left\|\left(u^{\varepsilon}-U^{\varepsilon}, v^{\varepsilon}-V^{\varepsilon}\right)\right\|_{H^{1}\left(\mathbb{R} \times \mathbb{R}_{+}\right)} \leq C
\end{gathered}
$$

$$
\left\|v^{\varepsilon}-f\left(u^{\varepsilon}\right)+a u_{x}^{\varepsilon}\right\|_{H^{1}\left(\mathbb{R} \times \mathbb{R}_{+}\right)} \leq C \varepsilon^{2} .
$$

\section{Limit}

In this section, we study the limiting behavior, as $\varepsilon$ goes to zero, of the solution $\phi_{z}^{\varepsilon}=\Phi^{\varepsilon}$ to (3.7), and then prove our main convergence theorem.

Proposition 5.1. Assume, in addition to the hypotheses of Theorem 4.1, that

(*) There exists $\tilde{\phi}_{0}$ such that $\Phi_{0}^{\varepsilon} \rightarrow \tilde{\phi}_{0}$ in $H^{2}$ as $\varepsilon \rightarrow 0$.

Then there exists an $\tilde{\phi}$ in $C^{1}([0, T] \times \mathbb{R})$ for any $T \geq 0$ such that

$$
\begin{gathered}
\Phi^{\varepsilon} \rightarrow \tilde{\phi} \quad \text { in } C^{0}\left([0, T] ; H_{l o c}^{2-\delta_{1}}\right) \quad \text { for any } \delta_{1}>0 \\
\Phi_{t}^{\varepsilon} \rightarrow \tilde{\phi}_{t} \quad \text { weakly in } L^{2}([0, T] \times \mathbb{R}),
\end{gathered}
$$

and $\tilde{\phi}$ satisfies the reduced equation

$$
\begin{gathered}
\tilde{\phi}_{t}-s \tilde{\phi}_{z}+f\left(U^{0}+\tilde{\phi}\right)_{z}-f\left(U^{0}\right)_{z}-a \tilde{\phi}_{z z}=0 \\
\left.\tilde{\phi}\right|_{t=0}=\tilde{\phi}_{0} .
\end{gathered}
$$

Proof. First, the sequence $\left(\phi_{z}^{\varepsilon}, \psi^{\varepsilon}\right)(t, z)$ satisfies the estimates

$$
\int_{0}^{t} \int_{-\infty}^{+\infty}\left\{\sum_{i=0}^{2}\left|\partial_{z}^{i}\left(\psi^{\varepsilon}, \phi_{z}^{\varepsilon}\right)\right|^{2}(\tau, z)+\sum_{i=0}^{1}\left|\partial_{z}^{i} \partial_{\tau}\left(\psi^{\varepsilon}, \phi_{z}^{\varepsilon}\right)\right|^{2}(\tau, z)\right\} d z d \tau \leq C
$$

and

$$
\left\|\phi_{z}^{\varepsilon}\right\|_{H^{1}\left(\mathbb{R} \times \mathbb{R}_{+}\right)} \leq C .
$$

Thus, $\left\{\Phi^{\varepsilon}\right\}=\left\{\phi_{z}^{\varepsilon}\right\}$ is in a bounded set of $C^{0}\left([0, T] ; H^{2}\right) \supset L^{2}\left([0, T] ; H^{2}\right)$ and $\left\{\Phi_{t}^{\varepsilon}\right\}$ is in a bounded set of $L^{2}\left([0, T] ; H^{1}\right)$. Hence, by the known compactness theorem, some 
sequence (still denoted) $\Phi^{\varepsilon}$ converges in $L^{2}\left([0, T] ; H_{l o c}^{1}\right)$ to some $\tilde{\phi}$ as $\varepsilon \rightarrow 0$. Also $\tilde{\phi} \in L^{\infty}\left([0, T] ; H^{2}\right)$ since $\sup _{0 \leq t \leq T}\left\|\Phi^{\varepsilon}\right\|_{2} \leq$ constant for all $\varepsilon$. Now,

$$
\begin{aligned}
& \left\|\Phi^{\varepsilon}-\tilde{\phi}\right\|_{C^{0}\left([0, T] ; H_{l o c}^{1}\right)} \leq C\left\|\Phi^{\varepsilon}-\tilde{\phi}\right\|_{H^{\frac{2}{3}}\left([0, T] ; H_{l o c}^{1}\right)} \\
& \quad \leq C\left\|\Phi^{\varepsilon}-\tilde{\phi}\right\|_{L^{2}\left([0, T] ; H_{l o c}^{1}\right)}^{1 / 3} \cdot\left\|\Phi^{\varepsilon}-\tilde{\phi}\right\|_{H^{1}\left([0, T] ; H_{l o c}^{1}\right)}^{2 / 3} \rightarrow 0 \quad \text { as } \quad \varepsilon \rightarrow 0
\end{aligned}
$$

since the first term on the right-hand side $\rightarrow 0$ and the second is bounded. Furthermore,

$$
\left\|\Phi^{\varepsilon}-\tilde{\phi}\right\|_{C^{0}\left([0, T] ; H_{l o c}^{2-\delta_{1}}\right)} \leq\left\|\Phi^{\varepsilon}-\tilde{\phi}\right\|_{C^{0}\left([0, T] ; H_{l o c}^{1}\right)}^{\delta_{1}} \cdot\left\|\Phi^{\varepsilon}-\tilde{\phi}\right\|_{C^{0}\left([0, T] ; H_{l o c}^{2}\right)}^{1-\delta_{1}},
$$

so $\Phi^{\varepsilon} \rightarrow \tilde{\phi}$ in $C^{0}\left([0, T] ; H_{l o c}^{2-\delta_{1}}\right)$.

Next, the boundedness of $\left\{\Phi_{t}^{\varepsilon}\right\}$ in $L^{2}\left([0, T] ; H^{1}\right) \subset L^{2}([0, T] \times \mathbb{R})$ implies that some subsequence (still denoted) $\Phi_{t}^{\varepsilon} \rightarrow$ some $w$ weakly in $L^{2}([0, T] \times \mathbb{R})$. In particular, for $\eta \in C_{0}^{\infty},(w, \eta) \leftarrow\left(\Phi_{t}^{\varepsilon}, \eta\right)=-\left(\Phi^{\varepsilon}, \eta_{t}\right) \rightarrow-\left(\tilde{\phi}, \eta_{t}\right)$, so $w=\tilde{\phi}_{t}$ in the sense of distributions. Once we show that $\tilde{\phi}$ satisfies (5.3)-(5.4) classically, then uniqueness for that system implies that the above convergences hold without restriction to a sequence since all sequences have subsequences converging to the same limit, and hence (5.1)-(5.2) hold.

On the other hand, since $U^{\varepsilon}-U^{0} \rightarrow 0$ in $H^{1}(\mathbb{R})$ and $f \in C^{3}$, we get

$$
f\left(\Phi^{\varepsilon}+U^{\varepsilon}\right)_{z}-f\left(\tilde{\phi}+U^{0}\right)_{z} \rightarrow 0 \text { in } L^{2}
$$

and

$$
f\left(U^{\varepsilon}\right)_{z}-f\left(U^{0}\right)_{z} \rightarrow 0 \text { in } L^{2} .
$$

Combining the above convergence results with Theorem 4.1, we conclude that $\psi^{\varepsilon}$ strongly converges in $H^{1}$

$$
\psi^{\varepsilon} \rightarrow f\left(U^{0}+\tilde{\phi}\right)-f\left(U^{0}\right)-a \tilde{\phi}_{z} \quad \text { in } \quad H^{1} .
$$

Taking $\varepsilon$ to zero in the equality

$$
\Phi_{t}^{\varepsilon}-s \Phi_{z}^{\varepsilon}+\psi_{z}^{\varepsilon}=0
$$

we have that the limit function $\tilde{\phi}$ satisfies

$$
\tilde{\phi}_{t}-s \tilde{\phi}_{z}+f\left(U^{0}+\tilde{\phi}\right)_{z}-f\left(U^{0}\right)_{z}-a \tilde{\phi}_{z z} \rightarrow 0
$$

weakly in $L^{2}([0, T] \times \mathbb{R})$ as $\varepsilon \rightarrow 0$. On the other hand, for any $\eta \in C_{0}^{\infty}$,

$$
\begin{aligned}
\left|\left(\eta, \Phi_{t}^{\varepsilon}-s \Phi_{z}^{\varepsilon}+f\left(U^{\varepsilon}+\Phi^{\varepsilon}\right)_{z}-f\left(U^{\varepsilon}\right)_{z}-a \Phi_{z z}^{\varepsilon}\right)\right| & =\left|\left(\eta, \varepsilon^{2}\left(\psi_{t}^{\varepsilon}-s \psi_{z}^{\varepsilon}\right)_{z}\right)\right| \\
& \leq \varepsilon\left[\left|\left(\eta_{t}, \varepsilon \psi_{z}^{\varepsilon}\right)\right|+s\left|\left(\eta, \varepsilon \psi_{z z}^{\varepsilon}\right)\right|\right] \leq C \varepsilon
\end{aligned}
$$

by (4.8). Since $C_{0}^{\infty}$ is dense in $L^{2}$, we have

$$
\tilde{\phi}_{t}-s \tilde{\phi}_{z}+f\left(U^{0}+\tilde{\phi}\right)_{z}-f\left(U^{0}\right)_{z}-a \tilde{\phi}_{z z}=0
$$

and assumption $(*)$ implies $\left.\tilde{\phi}\right|_{t=0}=\tilde{\phi}_{0}$. Finally, (5.1) implies that $\tilde{\phi} \in C^{0}\left([0, T] ; C^{1}\right)$ and then (5.3) shows that $\tilde{\phi} \in C^{1}([0, T] \times \mathbb{R})$. This completes the proof of Proposition 5.1 .

Now we turn to proving our main convergence theorem. 
Theorem 5.1. Suppose that the functions $\left(u^{\varepsilon}, v^{\varepsilon}\right)$ are solutions of the Cauchy problem $\left(E_{\varepsilon}\right)$ and $(1.5)$ uniquely determined by Theorem $4.2,\left(U^{\varepsilon}, V^{\varepsilon}\right)$ are traveling wave solutions of $\left(E_{\varepsilon}\right)$. If the initial data satisfy $\left(\mathrm{H}_{2}\right)$ and

$$
\begin{gathered}
u_{0}^{\varepsilon}-U^{\varepsilon} \rightarrow u_{0}-U^{0} \quad \text { in } \quad H^{2} \quad \text { as } \quad \varepsilon \rightarrow 0, \\
v_{0}^{\varepsilon}-V^{\varepsilon} \rightarrow v_{0}-V^{0} \quad \text { in } \quad H^{2} \quad \text { as } \quad \varepsilon \rightarrow 0
\end{gathered}
$$

and $\int_{R}\left(u_{0}^{\varepsilon}-u_{0}\right)(x) d x=0$, then there exists a $u \in C^{1}([0, T] \times \mathbb{R})$ for any $T \geq 0$ such that

$$
\begin{gathered}
u^{\varepsilon}-U^{\varepsilon} \rightarrow u(x, t)-U^{0} \quad \text { in } C^{0}\left([0, T] ; H_{\text {loc }}^{2-\delta_{1}}\right) \text { for any } \delta_{1}>0 \\
v^{\varepsilon}-V^{\varepsilon} \rightarrow v(x, t)-V^{0} \quad \text { in } C^{0}\left([0, T] ; H_{\text {loc }}^{2-\delta_{1}}\right)
\end{gathered}
$$

and the limit function $(u, v)$ satisfies the reduced equations

$$
\begin{aligned}
u_{t}+f(u)_{x} & =a u_{x x}, \\
\left.u\right|_{t=0} & =u_{0}(x)
\end{aligned}
$$

and

$$
v=f(u)-a u_{x} .
$$

Proof. The system $\left(\mathrm{E}_{\varepsilon}\right)$ can be expressed in terms of the perturbation $\left(\phi_{z}^{\varepsilon}, \psi^{\varepsilon}\right)=$ $\left(\Phi^{\varepsilon}, \psi^{\varepsilon}\right)$ and traveling waves $\left(U^{\varepsilon}, V^{\varepsilon}\right)$ defined in Section 2 as follows:

$$
\begin{gathered}
\phi_{z t}^{\varepsilon}-s \phi_{z z}^{\varepsilon}+\psi_{z}^{\varepsilon}+V^{\varepsilon}=0 \\
\varepsilon^{2}\left[\psi_{t}^{\varepsilon}-s \psi_{z}^{\varepsilon}\right]+a \phi_{z z}^{\varepsilon}=f\left(U^{\varepsilon}+\phi_{z}^{\varepsilon}\right)-f\left(U^{\varepsilon}\right)-\psi^{\varepsilon} .
\end{gathered}
$$

Here, we have used (2.1). Setting

$$
\psi=f\left(U^{0}+\tilde{\phi}\right)-f\left(U^{0}\right)-a \tilde{\phi}_{z}
$$

and applying the transformations

$$
\begin{gathered}
(z, t) \rightarrow(x-s t, t), \\
\tilde{\phi} \rightarrow u(x, t)-U^{0}(x-s t) \text { and } \psi \rightarrow v(x, t)-V^{0}(x-s t)
\end{gathered}
$$

in (5.6), we get

$$
u_{t}+f(u)_{x}-a u_{x x}+a U_{z z}^{0}+s U_{z}^{0}-f\left(U^{0}\right)_{z}=0
$$

and

$$
v(x, t)=V^{0}(z)+f(u)-f\left(U^{0}\right)-a u_{x x}+a U_{z z}^{0} .
$$

Since $\left(U^{0}, V^{0}\right)$ satisfies

$$
a U_{z z}^{0}+s U_{z}^{0}-f\left(U^{0}\right)_{z}=0, \quad V_{z}^{0}=s U_{z}^{0},
$$

we get

$$
u_{t}+f(u)_{x}=a u_{x x}, \quad v=f(u)-a u_{x x} .
$$

This ends the proof of Theorem 5.1. 
Acknowledgment. The first author was supported in part by the National Science Foundation grants DMS-9404157 and DMS-9704957, and the second author was supported in part by Natural Science Foundation of China and by the National Science Foundation grant INT-9601376. The second author thanks Professor M. Slemrod for useful conversations and constant encouragement. This work was begun while the second author was visiting the Georgia Institute of Technology, Atlanta, to which he is deeply grateful for its hospitality.

\section{References}

1. C. Bardos, F. Golse, and C.D. Levermore, Fluid dynamic limit of kinetic equations II: Convergence proofs for the Boltzmann equations, Comm. Pure Appl. Math. 46 (1993), 667-753.

2. A. Bensoussan, J.L. Lions, and G.C. Papanicolaou, Asymptotic Analysis for Periodic Structures, North-Holland, 1978.

3. C. Cercignani, The Boltzmann Equation and its Applications, Springer-Verlag, New York, 1988.

4. C. Cercignani, R. Illner, and M. Pulvirenti, The Mathematical Theory of Dilute Gases, SpringerVerlag, New York, 1994.

5. G. Q. Chen, C. D. Levermore, and T. P. Liu, Hyperbolic conservation laws with stiff relaxation terms and entropy, Comm. Pure. Appl. Math. 47 (1994), 787-830.

6. J.F. Collet and M. Rascle, Convergence of the relaxation approximation to a scalar nonlinear hyperbolic equation arising in chromatography, Z. Angew. Math. Phys. 47 (1996), 400-409.

7. L. Hsiao and T.P. Liu, Convergence to nonlinear diffusion waves for solutions of a system of hyperbolic conservation laws with damping, Comm. Math. Phys. 143 (1992), 599-605.

8. S. Jin and Z. P. Xin, The relaxation schemes for systems of conservation laws in arbitrary space dimensions, Comm. Pure. Appl. Math. 48 (1995), 235-277.

9. M.A. Katsoulakis and A.E. Tzavaras, Contractive relaxation systems and the scalar multidimensional conservation laws, Comm. PDE. 22 (1997), 195-233.

10. E.W. Larsen and J.B. Keller, Asymptotic solutions of the neutron transport problem, J. Math. Phys. 15 (1974), 75-81.

11. P.D. Lax, Hyperbolic Systems of Conservation Laws and the Mathematical Theory of Shock Waves, CBMS Reg. Conf. Series in Appl. Math. 11, SIAM, Philadelphia, 1973.

12. P.L. Lions and G. Toscani, Diffusive limit for finite velocity Boltzmann kinetic models, preprint (1996).

13. H.L. Liu and J. Wang, Asymptotic stability of traveling wave solutions of a hyperbolic system with relaxation terms, Beijing Mathematics 1997.

14. H.L. Liu, J. Wang, and T. Yang, Stability of a relaxation model with a nonconvex flux, to appear in SIAM J. Math. Anal.

15. T. P. Liu, Hyperbolic conservation laws with relaxation, Comm. Math. Phys. 108 (1987), 153175.

16. P. Marcati and A. Milani, The one-dimensional Darcy's law as the limit of a compressible Euler law, J. Diff. Eqn. 13 (1990), 129-147.

17. P. Marcati and B. Rubino, Hyperbolic to parabolic relaxation theory for quasilinear first order systems, preprint (1996).

18. R. Natalini, Convergence to equilibrium for the relaxation approximations of conservation laws, Comm. Pure Appl. Math. 49 (1996), 1-30.

19. __ A discrete kinetic approximation of entropy solutions to multidimensional scalar conservation laws, J. Diff. Eqn., to appear.

20. __ Recent mathematical results on hyperbolic relaxation problems, preprint (1998).

21. D. Serre, Relaxation semi-linèaire et cinètique des systèmes de lois de conservation, preprint (1998).

22. G. B. Whitham, Linear and Nonlinear Waves, Wiley, New York, 1974.

School of Mathematics, Georgia Institute of Technology, Atlanta, GA 30332, USA.

E-mail: jin@math.gatech.edu

Department of Mathematics, Henan Normal University, Xinxiang 453002, P.R. China. 